\title{
Transport Northbound Interface: The Need for Specification and Standards Coordination
}

\author{
D. King, C. Rotsos, University of Lancaster \\ I. Busi, F. Zhang, Huawei Technologies \\ N. Georgalas, British Telecom
}

\begin{abstract}
Next generation optical transport networks have high benchmarks for flexibility, reliability, and operational simplicity. These requirements underline a common, technologyindependent orchestration paradigm that can be extended to represent and configure specific optical technology attributes. Although, orchestration is an ongoing aspect of the current optical transport network evolution, the meaning and scope of orchestration is often only implied, and various Specification and Standards communities cannot always agree the requirements and objectives.

This paper describes the high-level requirements facing optical transport networks to provide well-defined Transport Northbound Interface (T-NBI) for optical resource programmability, control, and management automation. It explores the overall functionality that must be provided, whether encompassed in a single large-scale orchestration wrapper or partitioned into several sub-functions, of which only one component is designated as a transport orchestrator. It highlights the early efforts for optical transport resource modeling across Specification and Standardisation organisations.

The paper will report on recent Internet Engineering Task Force (IETF) Transport NBI Team Design Team efforts to collaborate across Standards Development Organisations (SDOs) to unify transport interface requirements and objectives. Finally, the paper will highlight use cases and applicability examples, and outline research gaps and challenges, opportunities for researchers, and areas for further collaboration between academia and industry.
\end{abstract}

Index Terms - Optical Modeling, Transport Northbound Interface (T-NBI), Transport Application Programming Interface (T-API).

\section{INTRODUCTION}

$\mathrm{T}$ RANSPORT Operator (Operator) infrastructure is comprised of multiple technologies across network layers (traffic engineered optical and packet). Typically, these resources are separated into multiple transport domains, each using different network technologies, control interfaces and implementing forwarding policy with diverse goals.

Management, configuration, and troubleshooting processes

The authors would also like to thank all members of the IETF Transport NBI Design Team involved in the definition of use cases, gap analysis and guidelines for using the IETF YANG models at the Northbound Interface (NBI) of a Transport SDN Controller. rely extensively on human intervention, using Element Management Systems (EMS) and Network Management Systems (NMS) to translate high-level connectivity goals into individual device configurations, while service deployment is designed using whiteboards by the network planners [1]. Correspondingly, transport service delivery times for new connections may take many months, with significant portions of this time spent in the design and configuration phase of the deployment life-cycle.

The inflexibility, and limited automation of Transport Networks, led to the development of new control and management architectures and protocols. We often to refer to this technology as Transport Software Defined Networking (TSDN): logically centralized control, separation of control and forwarding, open Application Programming Interface (API), and automation.

Existing optical transport networks often have separation of data plane and control elements; therefore, these are not new concepts, however establishing an open and well defined method for exposing transport capability via a Transport Northbound Interface (T-NBI), is now critical.

Potential success of Transport SDN in commercial environments is largely dependent on the success in specifying, documenting and standardising open transport interfaces between the Transport Orchestrator (T-O), Transport Controller (T-C) (Northbound Interface - NBI) and between TCs (EastWest Interface).

A common open interface to each boundary is pre-requisite for network operators to control multi-vendor and multi-domain networks also enable service provisioning coordination/automation. This must be achieved by using standardised models, used together with an appropriate messaging protocol (interface).

Several popular optical and transport SDN architectures and interfaces are being developed, including:

1. Generic functional architecture of transport networks [1], developed by the ITU Telecommunication Standardization Sector (ITU-T);

2. Transport-Application Programming Interface (T-API)

Furthermore, we would like to thank our colleagues at the ONF, MEF and ITU-T, and IETF for their ongoing support and cooperation of the transport NBI.

The corresponding author (Daniel King) is a Senior Research at the University of Lancaster - d.king@lancaster.ac.uk. 
Requirements [2] and Architecture [3], developed by the Open Networking Foundation (ONF);

3. Transport Northbound Interface Use Cases [3], Abstraction and Control of Traffic-Engineered Networks (ACTN) Framework [4], Traffic Engineering (TE) Topology [5] and TE Tunnel [6] YANG models defined by the Internet Engineering Task Force (IETF).

This document highlights the key components of control, interaction and naming of transport SDN functions, important use cases and requirements, and the type and scope of information that must be exchanged over the key interfaces.

\section{TRANSPORT SDN}

Transport network domains, including Optical Transport Network (OTN) and Wavelength Division Multiplexing (WDM) networks, are typically deployed based on a single vendor or technology platforms. They are often managed using proprietary interfaces to dedicated Element Management Systems (EMS), Network Management Systems (NMS) and increasingly Software Defined Network (SDN) controllers.

A well-defined open interface to each domain management system or controller is required for network operators to facilitate control automation and orchestrate end-to-end services across multi-domain networks. These functions may be enabled using standardized data models (e.g., YANG [7]), and appropriate messaging protocol (e.g., NETCONF (8)) or RESTCONF [9]) and encoding mechanisms.

\subsection{Transport Service Perspectives}

The following examples provide different use case perspectives for commercial transport SDN deployments.

1. End-to-End Service Management: Automated service creation covering Layer-0 to Layer-3.

2. Elastic Bandwidth Provisioning: Creation of elastic services with automatic or "on demand" changes in bandwidth.

3. Dynamic Datacenter Interconnections: Automatic load dependent fast service creation.

4. Transport as a Service (TaaS): Fully automate service requests including network planning and node configuration.

5. Multi-layer Network Operation: Multilayer optimized Layer-0 to Layer-3 networking with automatic setup and teardown.

6. Vendor Agnostic Transport Networking: Standardised transport SDN control interfaces for automated integration and deployment of services across multi-vendor equipment.

\subsection{Transport SDN Architecture}

The architecture of SDN is specified in the ONF SDN architecture document [3], which identifies core principles of SDN and applies them to transport networks.

The ACTN Framework [4] describes a control hierarchy and interfaces that would enable deployment of multi-domain Transport SDN networks.

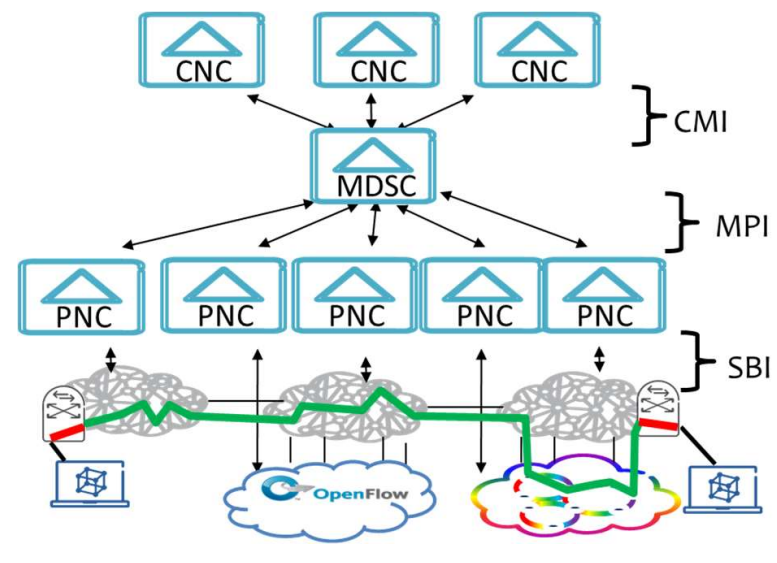

Fig. 1. IETF ACTN Control Hierarchy

The T-API Requirements [2] describes a functional architecture which has been used for the development of T-API requirements [2] and ongoing development of open source YANG modules.

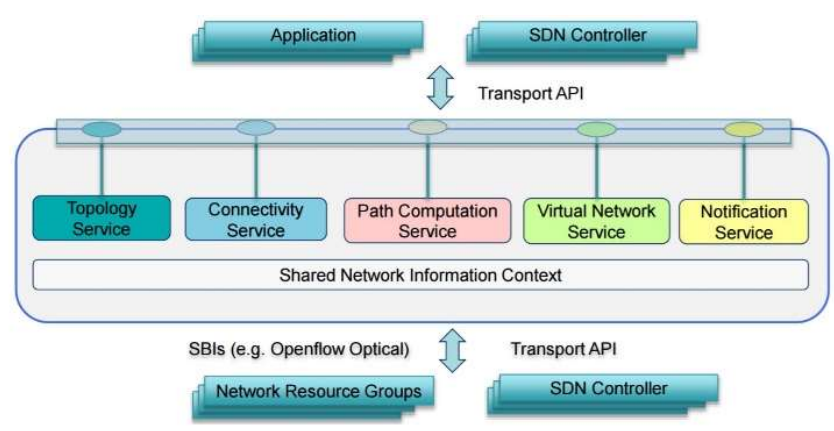

Fig. 2. ONF Transport API Functional Architecture

The underlying principles of these two reference architectures are very similar, but differences do exist.

An important design goal for application of these SDN principles to transport networks, is to be based SDN for transport on standardized and open interfaces at the northbound interface of the Transport SDN controller, to overcome the existing inter-operability limitations created by the lack of integration and interoperability of transport network devices.

Essentially, there is a clear need for a well-defined transport NBI and corresponding resource models. Combined, they are crucial for transport service orchestration, since they enable control and monitoring of service connectivity and network resource utilization and definition of custom fault management processes.

There are different opinions about whether this work would lead to interoperable and open resource models for the SBI; nevertheless, this work is complementary to the NBI definition, which would still be needed and it will also enable the integration of current deployments as well as a smooth migration of the transport network toward an open SBI paradigm, if it ever materializes. 


\subsection{Transport Service Orchestration}

Orchestration is a hot topic of current industry conversation dealing with network evolution. However, formal definitions do not exist, it remains an area where the meaning and scope of orchestration is often only implied.

Current understandings of orchestration include "the idea of automatically selecting resources to satisfy client demands" [10], which also defines orchestration as "The ongoing selection and use of resources by a provider to satisfy client demands according to optimization criteria." This definition is intended to encompass all the necessary aspects of a solution, while not compelling any subdivision of functionality, e.g., into intent or policy or network analytics (telemetry) which may be discussed separately.

Prior to SDN, transport devices supported many of Southbound Interfaces (SBI) protocols like Path Computation Element Protocol (PCEP), GMPLS, TL1, SNMP, CLI, XML, et al, which had been standardized but multi-vendor interoperable. With the advent of SDN and the use of centralized controllers to interface with transport devices, new transport devices are supporting also new protocols the SBI like NETCONF, OpenFlow, et al, making the southbound even more fragmented and still not multi-vendor interoperable. However, the application of SDN allows domain controllers to abstract the fragmented southbound view for its northbound clients by normalizing the NBI across various technologies, protocols, and vendors.

NBIs Would allow the transport domain controller to communicate with the orchestrator via the normalized NBI to automate and programmed end-to-end transport resources, leveraging the transport infrastructure in an optimized way, across single or multi-domain technologies, and multiple SBIs.

\subsection{Transport Northbound Interface}

Firstly, Northbound interfaces (NBIs) can be organized in two broad categories.

The first category contains low-level information modeling NBIs. The primary role of an information model is to converge state representation of data plane devices and abstract the heterogeneity of forwarding technology. Network information models have been developed before the introduction of the SDN paradigm by multiple formal and information SDOs, including the IETF and ITU-T.

Relevant to the SDN paradigm is the ONF information modeling working group (WG), which develops the Common Information Model (Core Model) specifications for a variety of interfaces, and not only the Transport NBI.

The Core Model is hierarchical and includes a central model, which provides a basic abstraction for data plane forwarding elements, and a technology forwarding and an application specific model, which evolve the core model abstraction. Core Model specifications exploit object inheritance and allow control applications to acquire abstract network connectivity information and, in parallel, access technology-specific attributes of network elements.

The second NBI category contains high-level and innovative control abstractions of the service request. These interfaces are typically implemented as SDN management applications, use the information model to implement their control logic and are consumed by external entities, like the OSS, the service orchestrator and other control applications.

Effectively, both interface types manifest themselves between the functional interfaces between the Network and Service Orchestrator components.

Any NBI will require resource models these are being developed in formal and informal SDOs, including: IETF, ONF and MEF; which can be used on the interfaces of a domain controller and an orchestrator. Each domain controller and orchestrator can use models developed by different SDOs. Therefore, it is important to ensure that all models support deployment use cases and related functionalities to allow a seamless translation and mediation between systems using different models.

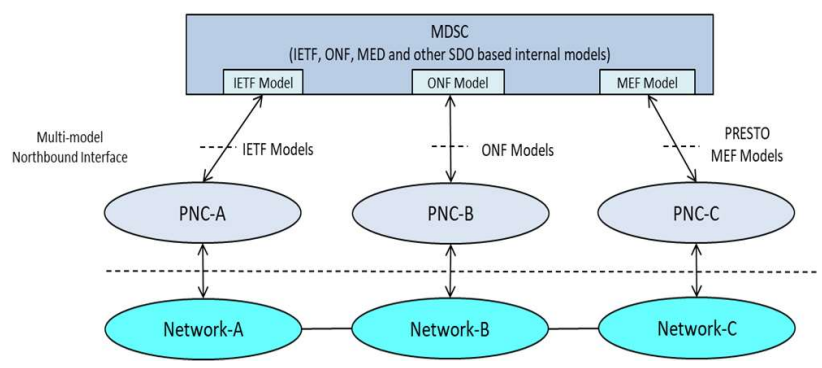

Fig. 3. IETF ACTN Applied YANG Models from multiple SDOs

\subsection{Defining the Transport Northbound Interface}

A transport network is a server-layer network designed to provide connectivity services, or more advanced services like Virtual Private Networks (VPN) for a client-layer network to carry the client traffic opaquely across the server-layer network resources. It acts as a pipe provider for upper-layer networks, such as IP network and mobile networks.

Transport networks, such as Synchronous Optical Networking (SONET) and Synchronous Digital Hierarchy (SDH), Optical Transport Network (OTN), Wavelength Division Multiplexing (WDM), and flexi-grid networks, are often built using equipment from a single vendor and are managed using private interfaces to dedicated Element Management Systems (EMS) and Network Management Systems (NMS). All transport networks have high benchmarks for reliability and operational simplicity. This suggests a common, technology-independent management and control paradigm that is extended to represent and configure specific technology attributes.

The need for operators to manage multi-vendor and multidomain transport networks (where each domain is an island of equipment from a single supplier) has been further stressed by the expansion in network size. At the same time, applications such as data center interconnection require larger and more dynamic connectivity matrices. Therefore, transport networks face new challenges going beyond automatic provisioning of tunnel setup enabled by GMPLS (Generalized Multi-Protocol Label Switching) protocols to achieve automatic service 
provisioning, as well as address opportunities enabled by partitioning the network through the process of resource slicing. With lower operational expenditure (OPEX) and capital expenditure (CAPEX) as the usual objectives, open interfaces to transport networks to meet these requirements. Again, the concept of SDN mentioned earlier leverages these ideas.

The YANG modeling language is the data modeling language of choice within the IETF and has been adopted by several industry-wide open management and control initiatives. YANG may be used to model both configuration and operational states; it is vendor-neutral and supports extensible APIs for control and management of elements.

There are several scenarios where an open interface to access transport network resources would be useful. For the data centre operator, assuming the objective is to trigger the transport network to provide connectivity on demand, the following capabilities, would typically be required for any "open" interface between multiple controllers:

- Acquisition of the topology, be it physical or logical, of the transport infrastructure resources;

- The ability to obtain information about a set of access points of the transport network facing the client side, including information such as access point identifiers, capabilities, location, and environment types (Data Centers, Storage, et al.);

- The capability to send a request for a service using the access point information, as well as the ability to retrieve a list of service requests and status: source nodes, destination nodes, and current bandwidth and service attributes;

- Telemetry and monitoring of network performance information for real-time monitoring and optimization.

Each of these capabilities will require management and control via open interfaces for multi-domain networks with homogeneous technologies (such as OTN), but it can be extended further to multi-domain networks with heterogeneous technologies with higher complexity.

\subsection{Core Requirements for the Transport Northbound Interface}

\subsubsection{Generic Requirements}

User Intent: Transport models should maintain separation between high-level user intent and the operational state of the network. For e.g., maintain separation between user service request, including all constraints, and the actual service and connection state in the network transport network.

State Management: Network and service objects should support the following states: administrative state, operational state, and lifecycle state. Administrative state and operational states are well understood. Lifecycle state is defined in the ONF to model the following entity lifecycle states: planned state, potential state, installed state, in conflict state, and pending removal state.

Identifiers: Network and service objects and would include a unique entity ID provided by the controller. The identifier would be chosen such that the same entity in a real network topology will always be identified through the same ID, even if the model is instantiated in separate data stores. Controllers may choose to capture semantics in the identifier, for example to indicate the type of entity and/or the type of the parent identity.

\subsubsection{Topology Requirements}

The model should support the following topological link and node definitions:

- $\quad$ Link Requirements

- Abstract Links

- Compound Link which are internally aggregated lower level links

- Access Links which connect the router port to the client port of the transport system

- $\quad$ Node Requirements

- Physical Node

- Abstract Node

- Chassis / Forwarding Domain

The Link should support various link related attributes including cost, latency, capacity, risk characteristics (including Shared Risk Link Groups - SRLGs). The model should provide clear association between Link and its topology (including virtual topology), nodes and termination points.

In cases of multi-layer networks, the model should be capable to provide information about the adaptation capability between layers within a network element. The model should also provide association between the Link and any underlay circuit or service supporting the Link.

\subsubsection{Telemetry Requirements}

Topology service clients (which in the Transport-SDN context could be various: applications, orchestrators, controllers, big data collectors, analytics processors, network planners, etc.) require accurate real time network state information (this is known as network telemetry).

Telemetry information will be instrumental for maintaining network efficiency and optimal control under failure conditions. Network telemetry streams would provide resource failure prediction across network resources and provide knowledge to route the provided transport connectivity services away from predicted failure areas; identify and predict points of congestion and eliminate and/or mitigate the congestion by deploying extra network capacity in a timely manner. Clearly network telemetry is a valuable source of information useful for network planning, troubleshooting and resource optimization, and will require suitable models, such as "YANG models for ACTN TE Performance Monitoring Telemetry and Network Autonomics" [11].

\section{TRANSPORT SERVICES}

Transport networks are generally designed to deal with "connections" or "services", which are entities that encompass multiple related optical forwarding technologies.

The transport orchestrator needs to be capable to request 
service connectivity from the transport controller to support application and/or IP routers connectivity. The type of services could depend of the type of physical links (e.g., OTN link, WSON link, ETH link or SDH link) between the routers and transport network.

\section{APPLICABILITY OF YANG TO TRANSPORT NORTHBOUND INTERFACE}

The transport NBI data models will required for representation of objects that can be configured or monitored within the transport system. Within the IETF, YANG [10] is the language of choice for documenting data models, and YANG models have been produced to allow configuration or modelling of a variety of network devices, protocol instances, and network services. YANG data models have been classified in [12] and for services in [13].

\section{CONCLUSION}

A variety of industry challenges remain for the development of standardised transport NBI. Emerging protocol and model solutions, as discussed in this paper, are immature and will require further investigation and development before they can be operationalised and used by operators.

The enabling SDOs for transport SDN need to work cooperatively, coordinated activities should include:

- Continued development of use cases and gap analysis [14], to identify a set of technology use cases and providing a gap analysis against existing transport models;

- Identify missing models: requirements for new models or where possible, augmentation of existing models;

- Providing guidelines, in terms of how all the related models, even when developed by different SDOs, may be used in a step-wise manner, these should be applied to network provider agreed transport network use cases;

- Finally, further research and investigation for network provider domain security and policy application and control, especially considering the inter-functional automation, should also be pursued.

\section{ACKNOWLEDGMENT}

The authors would also like to thank all members of the IETF Transport NBI Design Team involved in the definition of use cases, gap analysis and guidelines for using the IETF YANG models at the Northbound Interface (NBI) of a Transport SDN Controller.

Furthermore, we would like to thank our colleagues at the ONF, MEF and ITU-T for the ongoing support and cooperation of the transport NBI.

The authors are also grateful to the UK Engineering and Physical Sciences Research Council (EPSRC) for funding the TOUCAN project (EP/L020009/1), which supported much of the work presented in this paper.

\section{REFERENCES}

[1] International Telecommunications Union, "Generic functional architecture of transport networks", ITU-T Recommendation G.805, March 2000.

[2] ONF Technical Recommendation TR-527, "Functional Requirements for Transport API", June 2016.

[3] ONF Technical Recommendation TR-522 "SDN Architecture for Transport Networks", March 2016.

[4] D. Ceccarelli, Y. Lee, L. Fang, D. Lopez, S. Belotti, D. Dhody, D. King, "Framework for Abstraction and Control of Transport Networks", draft-ietf-teas-actn-framework, IETF Internet Draft, February 2017.

[5] Liu, X., Bryskin, I., Beeram, V., Saad, T., Shah, H., and O. Dios, "YANG Data Model for TE Topologies", draft-ietfteas-yang-te-topo, IETF Internet Draft, October 2016.

[6] Saad, T., Gandhi, R., Liu, X., Beeram, V., Shah, H., Bryskin, I., Chen, X., Jones, R., and B. Wen, "A YANG Data Model for Traffic Engineering Tunnels and Interfaces", draft-ietf-teas-yang-te, IETF Internet Draft, October 2016.

[7] Bjorklund, M., Ed., "The YANG 1.1 Data Modeling Language", IETF RFC 7950, August 2016.

[8] Bjorklund, M., "YANG - A Data Modeling Language for the Network Configuration Protocol (NETCONF)", IETF RFC 6020, October 2010.

[9] Bierman, A., Bjorklund, M., and K. Watsen, "RESTCONF Protocol", IETF RFC 8040, January 2017.

[10] ONF Technical Recommendation TR-540 "Functions of Orchestration", January 2017.

[11] Y. Lee, D. Dhody, S. Karunanithi, R. Vilalta, D. King, D. Ceccarelli, "YANG models for ACTN TE Performance Monitoring Telemetry and Network Autonomics", draft-leeteas-actn-pm-telemetry-autonomics, IETF Internet Draft, March 2017.

[12] Bogdanovic, D., Claise, B., and C. Moberg, "YANG Module Classification", draft-ietf-netmod-yang-modelclassification, IETF Internet Draft, October 2016.

[13] Zhang, X. and J. Ryoo, "A Service YANG Model for Connection-oriented Transport Networks", draft-zhang-teastransport-service-model, IETF Internet Draft, July 2016.

[14] I. Busi \& D. King, et al, "Transport Northbound Interface Use Cases”, draft-tnbidt-ccamp-transport-nbi-use-case, IETF Internet Draft, March 2017. 